\title{
Dynamics and Function of a CA1 Model of the Hippocampus during Theta and Ripples
}

\author{
Vassilis Cutsuridis` and Michael Hasselmo \\ Center for Memory and Brain, Boston University, Boston, MA, USA \\ vcut@bu.edu
}

\begin{abstract}
The hippocampus is known to be involved in spatial learning in rats. Spatial learning involves the encoding and replay of temporally sequenced spatial information. Temporally sequenced spatial memories are encoded and replayed by the firing rate and phase of pyramidal cells and inhibitory interneurons with respect to ongoing network oscillations (theta and ripples). Understanding how the different hippocampal neuronal classes interact during these encoding and replay processes is of great importance. A computational model of the CA1 microcircuit [3], [4, 5] that uses biophysical representations of the major cell types, including pyramidal cells and four types of inhibitory interneurons is extended to address: (1) How are the encoding and replay (forward and reverse) of behavioural place sequences controlled in the CA1 microcircuit during theta and ripples? and (2) What roles do the various types of inhibitory interneurons play in these processes?
\end{abstract}

Keywords: Computational model, microcircuit, inhibitory interneurons, STDP, calcium, theta, ripples, medial septum, CA1.

\section{Introduction}

Spatial memories in the hippocampus are encoded (stored) and replayed by the firing frequency and spike timing of pyramidal cells and inhibitory interneurons during network oscillations. Theta oscillations (4-10 Hz) are observed in rats during exploration and rapid eye movement (REM) sleep, whereas sharp waveassociated ripples $(100-200 \mathrm{~Hz})$ are observed during immobility, slow-wave sleep (SWS) and consummatory behaviours. During exploration hippocampal place cells have been shown to systematically shift their firing phase with respect to theta as the animal transverses the place field (a phenomenon known as phase precession) [19.

Many theories have been proposed over the years trying to understand how memories in the hippocampus are encoded and replayed during network oscillations [7, 15]. Buzsaki's two-stage memory model [15] hypothesized that both theta and sharp-wave (ripple) states of the hippocampus are essential to memory trace encoding and replay. During theta (exploratory behavior) neocortical

\footnotetext{
^ Corresponding author.
} 
A

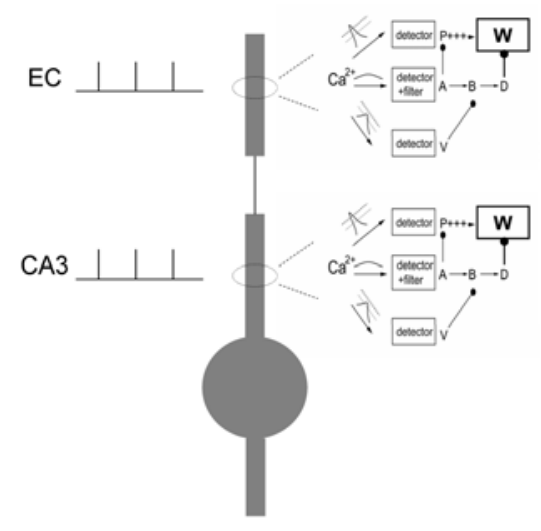

B

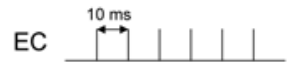

CA3

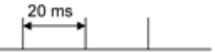

C

CA3

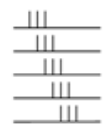

D

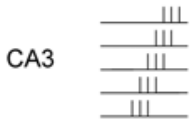

Fig. 1. (A) Pyramidal cell model with calcium detectors in distal and proximal dendrites. (B) Entorhinal cortical (EC) and Schaffer collateral (CA3) inputs during theta rhythm. (C) Forward replay of CA3 spatial memories used as inputs to CA1 during ripple activity (location $\mathrm{A}$ in figure 2). (D) Reverse replay of CA3 spatial memories used as inputs to CA1 during ripple activity (location B in figure 2).

information is transmitted to the hippocampus via the dentate gyrus, where it is encoded by pyramidal cells via synaptic plasticity mechanisms. During the sharpwave associated ripple state the pyramidal cells initiate population bursts, which then cause the already stored memories in the hippocampus to reach the neocortex and hence to be replayed. Hasselmo's and colleagues' oscillatory model [7] hypothesized that hippocampal theta rhythm (4-7 Hz) can contribute to memory formation by separating encoding (storage) and retrieval of memories into independent functional sub-cycles. Recent experimental evidence has shown that in the CA1 area of the hippocampus the same set of excitatory and inhibitory cells, which fire at specific phases during theta, are active at completely different phases during ripples [10], 11], 12]. Similarly, medial septal GABAergic neurons differentially phase their activities with respect to theta and ripple [14, [17.

Here we investigate, via computer simulations, the biophysical mechanisms by which encoding and replay of behaviourally relevant spatial memory sequences are achieved by the CA1 microcircuitry. A model of the CA1 microcircuit [3], 4], [5], 6] is extended that uses simplified biophysical representations of the major cell types, including pyramidal cells (PCs) and four types of inhibitory interneurons: basket cells (BCs), axo-axonic cells (AACs), bistratified cells (BSCs) and oriens lacunosum-moleculare (OLM) cells. Inputs to the network come from the entorhinal cortex (EC), the CA3 Schaffer collaterals and medial septum (MS). Our model addresses three important issues: (1) How is the mechanism of phase precession of place cells in the CA1 microcircuit achieved in presence of various types of inhibitory interneurons? (2) How are the encoding and replay (forward and reverse) of behavioural place sequences controlled in the CA1 microcircuit 
during theta and ripples? and (3) What roles do the various types of inhibitory interneurons play in these processes?

\section{Model Architecture and Properties}

The CA1 network model consisted of 4 pyramidal cells and four types of inhibitory interneurons: two basket cells, an axoaxonic cell, a bistratified cell and an oriens lacunosum-moleculare (OLM) cell. Hodgkin-Huxley mathematical formalism was used to describe the ionic and synaptic mechanisms of all cells. All simulations were performed using XPPAUT [20] running on a PC under windows XP. The biophysical properties of each cell were adapted from cell types reported in the literature [5], 9].

Pyramidal Cells. Each pyramidal cell consisted of 4 compartments: an axon, a soma, a proximal dendrite and a distal dendrite. Active properties included a fast $\mathrm{Na}^{+}$current, a delayed rectifier $\mathrm{K}^{+}$current, an LVA L-type $\mathrm{Ca}^{2+}$ current, an A-type $\mathrm{K}^{+}$current, and a calcium activated $\mathrm{mAHP} \mathrm{K}^{+}$current. No recurrent connections between pyramidal cells in the network were assumed.

Each pyramidal cell received proximal and distal excitation (AMPA and NMDA) from the CA3 Schaffer collaterals and entorhinal cortex (EC), respectively, and synaptic inhibition $\left(\mathrm{GABA}_{A}\right)$ from the BC, AAC, BSC, and OLM cells in the soma, axon, proximal dendrite and distal dendrite, respectively.

A mechanism for spike timing dependent plasticity (STDP) in each dendrite was used to measure plasticity effects. The mechanism had a modular structure consisting of three biochemical detectors, which responded to the instantaneous calcium level and its time course in the dendrite [1]. The detection system consisted of: (1) a potentiation (P) detector which detected calcium levels above a high-threshold (e.g. $4 \mu \mathrm{M}$ ) and triggered LTP, (2) a depression (D) detector which detected calcium levels exceeding a low threshold level (e.g. $0.6 \mu \mathrm{M}$ ), remained above it for a minimum time period and triggered LTD, and (3) a veto (V) detector which detected levels exceeding a mid-level threshold (e.g. $2 \mu \mathrm{M}$ ) and triggered a veto to the $\mathrm{D}$ response. Calcium entered the neuron through: (1) voltage-gated calcium channels (VGCCs), and (2) NMDA channels located at each dendrite. Calcium influx from neither channels alone elicited plasticity. Plasticity resulted only from the synergistic action of the two calcium sources (NMDA and VGCC). A graphical schematic of the model pyramidal cell and its calcium detectors for STDP is shown in Figure 1A.

Inhibitory Interneurons. All inhibitory interneurons consisted of a single compartment (soma). Active properties of BC, AAC and BSC included a fast $\mathrm{Na}^{+}$, a delayed rectifier $\mathrm{K}^{+}$and a type-A $\mathrm{K}^{+}$currents [5]. Active properties of the OLM cell included a fast $\mathrm{Na}^{+}$current, a delayed rectifier $\mathrm{K}^{+}$current, a persistent $\mathrm{Na}^{+}$current and an h-current 9]. During theta, axoaxonic and basket cells received excitatory inputs from the EC perforant path and the CA3 Schaffer 


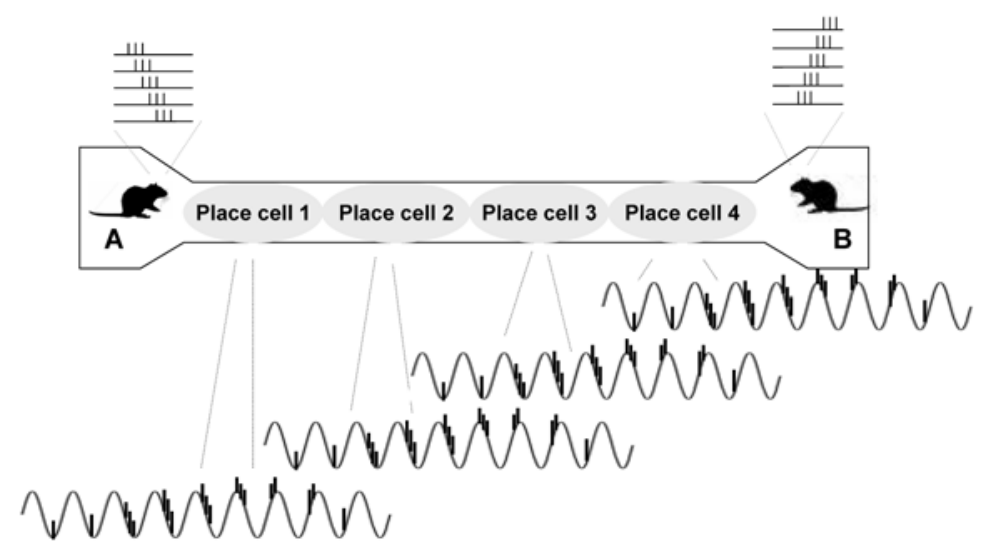

Fig. 2. Virtual linear track paradigm used. The rat must transverse the track starting from location A and stopping at location B. Gray filled ellipses represent the place fields (PF) of three pyramidal cells (PCs) in the network. Note their fields are nonoverlapping. As the rat transverses the $\mathrm{PF}$, each $\mathrm{PC}$ shifts its firing to earlier phases of the theta rhythm. At locations $\mathrm{A}$ and $\mathrm{B}$ the rats retrieve either the track locations to be transversed or the track locations already transversed, respectively.

collateral, inhibition from the medial septum, and recurrent excitation from the pyramidal cells. Basket cells recurrently inhibited each other and received additional inhibition from the bistratified cells. Bistratified cells were excited by the CA3 Schaffer collateral input only, inhibited by the medial septum, synaptically excited by $\mathrm{PC}$ recurrent excitation and synaptically inhibited by the basket cell. OLM cells received recurrent excitation from the PCs and forward inhibition from the medial septum. During ripples, the AAC, BC and BSC were excited only by the CA3 Schaffer collaterals and inhibited by the MS cells (see MODEL INPUTS subsection for details). The OLM cell was excited by the PCs and inhibited by the MS cells (see MODEL INPUTS subsection for details).

Model Inputs. Excitatory inputs (spikes) to network cells originated from the entorhinal cortex (EC) and CA3 Schaffer collaterals, whereas external inhibitory one from the medial septum (MS). During theta, the EC and CA3 inputs were continuously present, but at different frequencies (see Figure 1B). The interspike interval of the EC input was set to $10 \mathrm{~ms}(100 \mathrm{~Hz})$, whereas the ISI of the CA3 input was set to $20 \mathrm{~ms}(50 \mathrm{~Hz})$ [22]. Both EC and CA3 inputs arrived at the same time in the CA1-PC dendrites. The MS inputs were modelled as burst cells, which fired at specific phases of the theta rhythm. One MS burst cell fired at the peak of the extracellular theta (type 1) [14, whereas the other one at its trough (type 2) [17. MS cells inhibited only the network inhibitory interneurons. 
During ripples, the CA1 PCs received forward or reversed excitatory rippled input only from the CA3 Schaffer collaterals (see Figure 1C and 1D) [15], 21]. The inhibitory inputs from the MS cells were of two types: (1) a cell with a thetalike oscillation during the ripple-centered epoch, pausing its activity before the ripple peak and increasing its firing right after the ripple peak (type 1) [17, and (2) a cell that paused its activity during the ripple episode (type 2) [17.

\section{Results}

\subsection{Virtual Linear Track}

Our virtual linear track consisted of a rat running from station A to station B (see Figure 2). In stations $\mathrm{A}$ and $\mathrm{B}$ the rat was allowed to stand still awaiting for the GO signal to transverse the linear track. The linear track consisted of four non-overlapping place field representations of equal dimensions $(\approx 25 \mathrm{~cm})$. The virtual rat took $2.25 \mathrm{sec}$ (9 theta cycles) to tranverse through one place field. Figure 4 shows the firing activities of two place cells (PC 1 and PC4) and all inhibitory interneurons in two place fields (PF1 and PF4 in Figure 2). Each place field was encoded by the firing of a single pyramidal cell, whose phase of firing shifted with respect to the external theta rhythm 19. As the rat entered a place field (first theta cycle) of a given pyramidal cell, the first spikes occured close to the trough of the theta cycle. As the rat was approaching the end of the field (last theta cycle), they occured near the peak of the cycle, having precessed almost 180 degrees over the course of 9 theta cycles (see figure 4) 23]. This was accomplished by the constantly increasing strength of the proximal synapses due to the STDP learning rule, which increased the tendency of PCs to fire at earlier theta phases in the presence of a constant level inhibitory threshold (BSC inhibition) (simulation result not shown). Once the rat reached station B, it was rewarded and allowed to be engaged into consummatory behaviours. At the stations $\mathrm{A}$ and $\mathrm{B}$, our hippocampal simulation entered a different state of waking without theta rhythmic oscillations, where sharp wave associated ripple activity dominated the input and output of CA1 [16], 18. As experimental studies have shown at station A the rat experienced forward replay of neural activity coding the track locations to be transversed [16, whereas at station $\mathrm{B}$ the rat experienced reverse replay of neural activity coding the track locations it has just tranversed [18].

\subsection{Encoding of Spatiotemporal Memories during Theta}

Figure 3 depicts the encoding process of spatiotemporal memories during theta. During theta, input from EC enters the distal dendrite of the CA1 PC cells, whereas input from CA3 Schaffer collaterals enters the proximal dendrite of the PC cells. On their own, the EC inputs generate dendritic spikes in the distal dendrites, which get attenuated on their way to the soma (see figure 3A) 2]. The CA3 inputs generate excitatory postsynaptic potentials (EPSPs), which fail to 
A

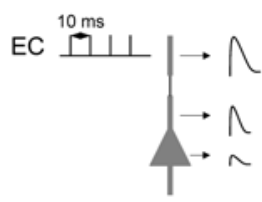

B

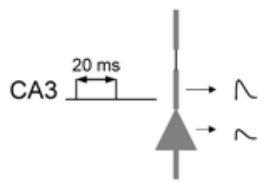

C

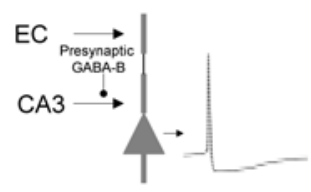

$\mathbf{F}$

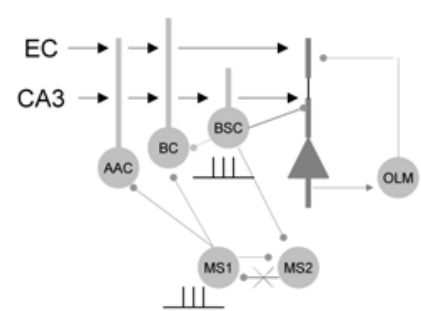

Fig. 3. Schematic of encoding of spatiotemporal memories during the theta rhythm (see text for details)
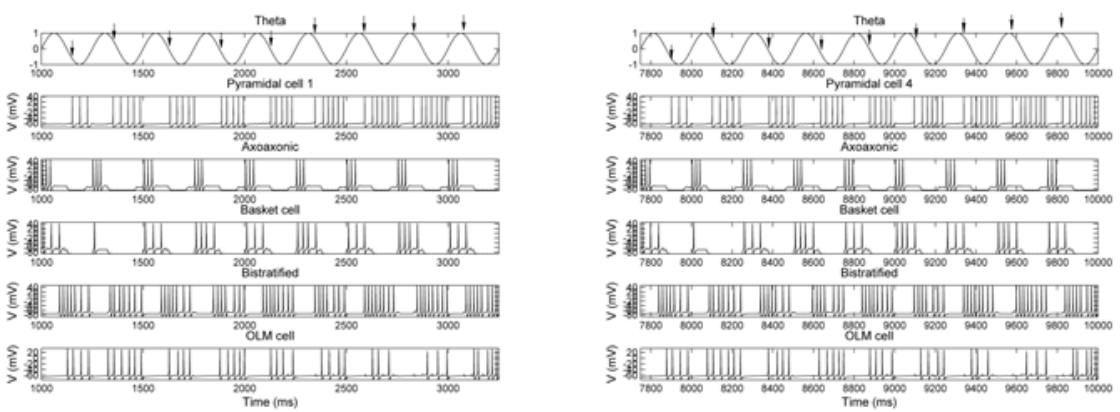

Fig. 4. (Left) Firing activities of pyramidal cell 1 and all inhibitory interneurons with respect to theta rhythm. (Right) Firing activities of pyramidal cell 4 and all inhibitory interneurons with respect to theta rhythm. Vertical arrows indicate phase precession of pyramidal cell firings in every theta cycle. Nine theta cycles comprise a pyramidal cell's (place cell's) place field.

generate somatic action potentials, because they are presynaptically inhibited by $\mathrm{GABA}_{B}$ (see figure 3B) [8. The presynaptic $\mathrm{GABA}_{B}$ inhibition in CA1 cyclically changed its strength with respect to theta (active during the first half of theta, inactive during the second half) 8]. During the first half of theta, the strength of CA3 input to PC proximal dendrites was reduced by $50 \%$. When the EC and CA3 inputs were concident in the proximal dendrites of the PCs, then action potentials are generated in the $\mathrm{PC}$ somas (figure $3 \mathrm{C}$ ). 
Figure 3D-F depicts which cells are active during theta. Figure 4 depicts the firing activities of all network cells during theta. During the first half-cycle of theta (0-180 degrees), we propose the following: the coincident EC and CA3 inputs cause first the AAC to fire action potentials with interspike interval (ISI) equal to 20ms, which inhibit the PCs at their axons and prevent them from firing APs (Figure 3D) [11]. Once the AAC stops firing, BCs, which are modeled as slow integrators [11], start to fire due to the coincident EC and CA3 inputs to their somas. Because of their mutual recurrent inhibition, each BC fires every $40 \mathrm{~ms}$ as in 11 . The role of BCs is to inhibit the PCs and prevent them from firing, pace subthreshold theta oscillations in PCs [13] and prevent the BSC from ruining learning in the PC proximal dendrite by inhibiting it (Figure 3E). BSC, along with the OLM cell, is also inhibited by the type 2 MS cell (Figure 3E). The type $2 \mathrm{MS}$ cell also inhibits the type $1 \mathrm{MS}$ cell, which in turn disinhibits the $\mathrm{AAC}$ and BCs and allows them to fire and carry-on with their inhibitory duties (Figure 3E).

Figure $3 \mathrm{~F}$ depicts the second sub-cycle of theta, which begins as the presynaptic GABA $_{B}$ inhibition to CA3 Schaffer collateral input to PC synapses declines and type 1 MS cell approach maximum activity. Because of this septal input, the basket and axoaxonic cells are now inhibited, releasing pyramidal cells, bistratified cells and OLM cells from inhibition. Pyramidal cells may now fire more easily, thus, allowing previously learned memories to be recalled. Type $1 \mathrm{MS}$ cell also inhibits the type $2 \mathrm{MS}$ cell, which in turn disinhibits the BSC and OLM cell. To ensure the correct place memory of the sequence is recalled, the disinhibited BSC broadcasts to all PCs a non-specific inhibitory signal, which allows the PCs that learned the place memory to recall it, while quenching all other spurious places memories (e.g. subsequent memories in the sequence). The OLM cell, which gets activated by the PCs, in turn send an inhibitory signal to the distal PC dendrite, which prevents the EC input from interfering with the recall of the pattern.

\subsection{Forward and Backward Replay of Memories during Ripple Activity}

Figure 5 depicts the replay processes during a ripple episode. In contrast to theta (figure 4), during the ripple episode (forward or reverse) the firing patterns of inhibitory interneurons in the network change (figure 6) [11], [12. During ripple activity what was locally learned during theta oscillations, it is now retrieved by the subiculum, entorhinal cortex and the neocortex, where it reached consciousness [15]. The synaptic weights of the proximal dendrite are now fixed (no changing). Forward and reverse replay activity arise from the highly synchronous activity of the CA3 PCs [15], 21]. This highly synchronous activity excites first the AAC, which is disinhibited by the type $1 \mathrm{MS}$ cell (as we mentioned before the type $1 \mathrm{MS}$ cell pauses its activity for about $25 \mathrm{~ms}$ before the peak of the ripple episode and increases it right after it), and in turn inhibit the axons of all CA1 PCs in the network. The duration of this axonal inhibition is short (less than 


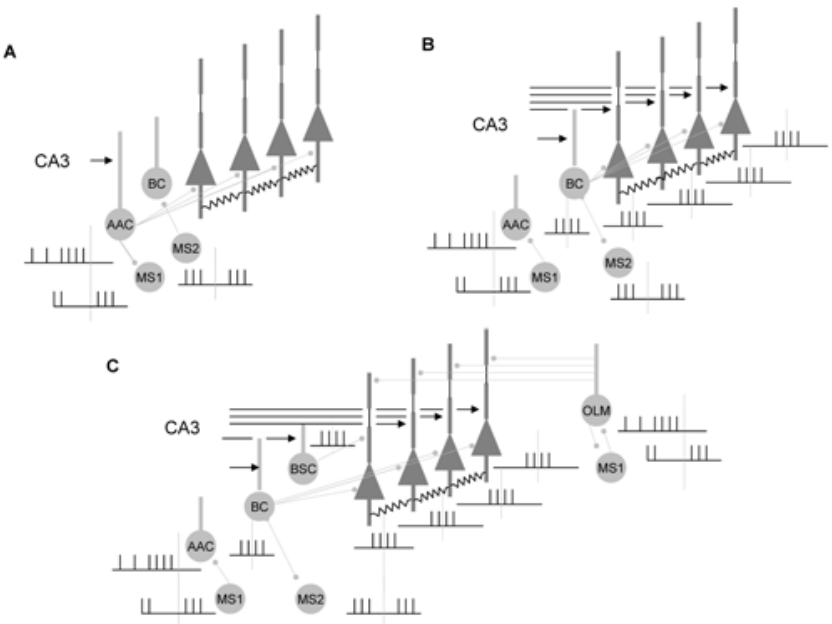

Fig. 5. Schematic of the forward and backward replay processes of spatiotemporal memories during ripple activity (see text for details). Vertical dashed gray line indicates the peak of the ripple episode.
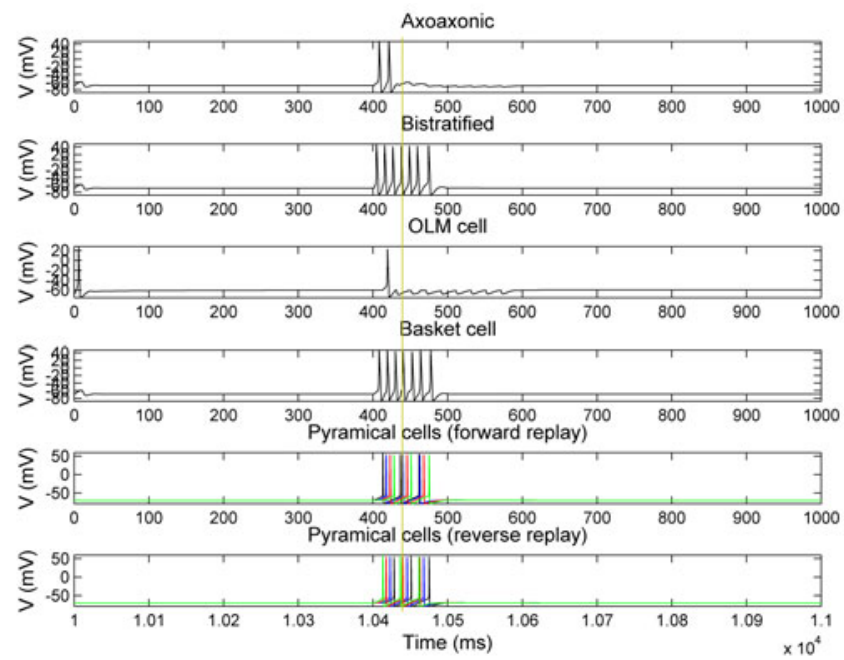

Fig. 6. Firing activities of all network cells with respect to ripples during both forward and reverse replay of spatiotemporal memories. Dashed yellow line indicates peak amplitude of ripple episode. Axoaxonic, basket, bistratified and OLM cells fire the same way during both forward and reverse replay of memories [11, 12. During forward replay, pyramidal cells fire in a specific order: black, blue, red, and green. During reverse replay the order is reversed: green, red, blue, and black. 
$25 \mathrm{~ms}$ ), since the AAC pauses its firing right after the peak of ripple episode due to increased activity of the type $1 \mathrm{MS}$ cell. The role of the AAC is to silence the CA1 network and prepare it for the appropriate retrieval of information based on the current context. Similar firing activity has the OLM cell in the network, because it is also (dis)inhibited by a type $1 \mathrm{MS}$ cell. The role of the OLM cell is to prevent the EC input from interfering with the recall of the memory.

Next, BCs and BSC, which were inhibited by the type 2 MS cells during the start of the ripple episode are now disinhibited by them and become active from the highly synchronous CA3 input. The role of the $\mathrm{BSC}$ is to provide a nonspecific inhibition to all PCs, allowing this way only the "appropriate" PCs that learned the pattern(s) to recall it(them). The role of the BCs is to maintain the highly syncronous ripple activity of the PCs. A similar role is played by the gap channels in the PC axons [24].

\section{Conclusion}

A reduced version of a previously published CA1 microcircuit model [3, 4, [5], 6] is extended to simulate how spatiotemporal patterns are encoded and retrieved in the CA1 area of the hippocampus during theta and ripples. Our model demonstrates: (1) How is phase precession of place cells in CA1 achieved in the presence of various types of inhibitory interneurons? and (2) How are encoding and replay (forward and reverse) of behavioural sequences of spatial memories controlled in CA1 during theta and ripples in the presence of various types of inhibitory interneurons. Much more work is needed to further explore why the model place cells don't precess beyond 180 degrees with respect to theta. Does a full 360 degrees phase precession result from the interactions of a larger and noiser network of cells or is it driven by phase precessed CA3 inputs? How does a larger number of simulated theta cycles contribute to phase precession? Also, what happens to network dynamics and its ability to learn when the presentation frequencies of inputs change? Finally, what role does dopamine in CA1 play in binding together temporally sequenced spatial memories?

Acknowledgement. This work was funded by NSF Science of Learning Center CELEST grant SMA 0835976, NIMH R01 MH61492, NIMH R01 MH60013 and NIMH Silvio Conte Center grant P50 MH71702.

\section{References}

[1] Rubin, J.E., Gerkin, R.C., Bi, G.Q., Chow, C.C.: Calcium time course as signal for spike-timing-dependent plasticity. J. Neurophysiol. 93, 2600-2613 (2005)

[2] Jarsky, T., Roxin, A., Kath, W.L., Spruston, N.: Conditional dendritic spike propagation following distal synaptic activation of hippocampal CA1 pyramidal neurons. Nat. Neurosci. 8(12), 1667-1676 (2005) 
[3] Cutsuridis, V., Cobb, S., Graham, B.P.: Encoding and Retrieval in a CA1 Microcircuit Model of the Hippocampus. In: Kurkova, V., et al. (eds.) ICANN 2008, Part II. LNCS, vol. 5164, pp. 238-247. Springer, Heidelberg (2008)

[4] Cutsuridis, V., Cobb, S., Graham, B.P.: Hippocampus, microcircuits and associative memory. Neural Networks 22(8), 1120-1128 (2009)

[5] Cutsuridis, V., Cobb, S., Graham, B.P.: Encoding and retrieval in the hippocampal CA1 microcircuit model. Hippocampus 20(3), 423-446 (2010)

[6] Graham, B.P., Cutsuridis, V., Hunter, R.: Associative Memory Models of Hippocampal Areas CA1 and CA3. In: Cutsuridis, V., et al. (eds.) Hippocampal Microcircuits: A Computational Modeller's Resource Book, pp. 461-494. Springer, Heidelberg (2010)

[7] Hasselmo, M., Bodelon, C., Wyble, B.: A proposed function of the hippocampal theta rhythm: separate phases of encoding and retrieval enhance reversal of prior learning. Neural Comput. 14, 793-817 (2002)

[8] Molyneaux, B.J., Hasselmo, M.: GABA $B$ presynaptic inhibition has an in vivo time constant sufficiently rapid to allow modulation at theta frequency. J. Neurophys. 87(3), 1196-1205 (2002)

[9] Kunec, S., Hasselmo, M.E., Kopell, N.: Encoding and retrieval in the CA3 region of the hippocampus: a model of theta-phase separation. J. Neurophysiol. 94(1), 70-82 (2005)

[10] Klausberger, T., Somogyi, P.: Neuronal diversity and temporal dynamics: the unity of hippocampal circuit operations. Science 321, 53-57 (2008)

[11] Klausberger, T., Magill, P.J., Marton, L.F., David, J., Roberts, B., Cobden, P.M., Buzsaki, G., Somogyi, P.: Brain-state- and cell-type-specific firing of hippocampal interneurons in vivo. Nature 421, 844-848 (2003)

[12] Klausberger, T., Marton, L.F., Baude, A., Roberts, J.D., Magill, P.J., Somogyi, P.: Spike timing of dendrite-targeting bistratified cells during hippocampal network oscillations in vivo. Nat. Neurosci. 7(1), 41-47 (2004)

[13] Cobb, S.R., Buhl, E.H., Halasy, K., Paulsen, O., Somogyi, P.: Synchronization of neuronal activity in hippocampus by individual GABAergic interneurons. Nature 378(6552), 75-78 (1995)

[14] Borhegyi, Z., Varga, V., Szilagyi, N., Fabo, D., Freund, T.F.: Phase segregation of medial septal GABAergic neurons during hippocampal theta activity. J. Neurosci. 24(39), 8470-8479 (2004)

[15] Buzsaki, G.: Two stage model of memory trace formation: a role for "noisy" brain states. Neurosci. 31(3), 551-570 (1989)

[16] Diba, K., Buzsaki, G.: Forward and reverse hippocampal place-cell sequences during ripples. Nat. Neurosci. 10(10), 1241-1242 (2007)

[17] Dragoi, G., Carpi, D., Recce, M., Csicsvari, J., Buzsaki, G.: Interactions between hippocampus and medial septum during sharp waves and theta oscillation in the behaving rat. J. Neurosci. 19(14), 6191-6199 (1999)

[18] Foster, D.J., Wilson, M.A.: Reverse replay of behavioural sequences in hippocampal place cells during the awake state. Nature 440, 680-683 (2006)

[19] O'Keefe, J., Recce, M.L.: Phase relationship between hippocampal place units and the EEG theta rythm. Hippocampus 3(3), 317-330 (1993)

[20] Ermentrout, B.: Simulating, analyzing, and animating dynamical systems: A guide to XPPAUT for researchers and students. SIAM, Philadelphia (2002)

[21] Koene, R.A., Hasselmo, M.E.: Reversed and forward buffering of behavioral spike sequences enables retrospective and prospective retrieval in hippocampal regions CA3 and CA1. Neural Networks 21, 276-288 (2008) 
[22] Colgin, L.L., Denninger, T., Fyhn, M., Hafting, T., Bonnevie, T., Jensen, O., Moser, M.B., Moser, E.I.: Frequency of gamma oscillations routes flow of information in the hippocampus. Nature 462(19), 353-358 (2009)

[23] Schmidt, R., Diba, K., Leibold, C., Schmitz, D., Buzsaki, G., Kempter, R.: Singletrial phase precession in the hippocampus. J. Neurosci. 29(42), 13232-13241 (2009)

[24] Traub, R.D., Draguhn, A., Whittington, M.A., Baldeweg, T., Bibbig, A., Buhl, E.H., Schmitz, D.: Axonal gap junctions between principal neurons: a novel source of network oscillations, and perhaps epileptogenesis. Rev. Neurosci. 13, 1-30 (2002) 